\title{
A Curious Case of Unresolving Pneumonia
}

\author{
Kilaru D*, Anand P and Chua W
}

Department of Internal Medicine, Nassau University Medical Center, East Meadow, New York, USA

\begin{abstract}
Granulomatosis with Polyangiitis (GPA), formerly known as Wegener's Granulomatosis, is a rare multisystem autoimmune disease of unknown etiology. GPA is characterized by necrotizing granulomatous inflammation and pauciimmune vasculitis in small- and medium-sized blood vessels. Given its protean clinical manifestations, the diagnosis of GPA can be difficult to distinguish from infectious etiologies. Here we describe a case of GPA diagnosed based on a positive ANCA serology and endobronchial features which responded to treatment with only steroids.
\end{abstract}

Keywords: Pneumonia; Granulomatosis; Respiratory distress

\section{Introduction}

Granulomatosis with Polyangiitis (GPA), formerly known as Wegener's Granulomatosis, is a rare multisystem autoimmune disease of unknown etiology. Given its protean clinical manifestations, the diagnosis of GPA can be difficult to distinguish from infectious etiologies. Here we describe a case of GPA diagnosed based on a positive Ani serology and endobronchial features which responded to treatment with only steroids.

\section{Case Presentation}

A 32-year-old African American female presented with syncope after a coughing fit. The patient complained of an intermittent cough with blood tinged whitish sputum for one month and was associated with shortness of breath that usually occurs with a bout of coughing. One week prior to presentation she experienced fever, chills, ear pain and flu like symptoms and completed levofloxacin course prior to admission with no improvement in symptoms. Patient denied any night sweats, weight loss, insect bites, rashes or sick contacts. She travelled to Jamaica 1 year ago. No significant past medical history, allergies and family history. She denied smoking and drugs. She worked as a dental assistant [1-4].

On Physical Examination, the patient was in mild respiratory distress with a temperature of 101, Pulse of 110 and, oxygen saturation of $99 \%$ on room air. She had tender submandibular and supraclavicular lymphadenopathy. Nasal turbinates showed no lesions. Pulmonary auscultation revealed right upper and mid lung field crackles. Laboratory examination revealed a predominantly neutrophilic leukocytosis with

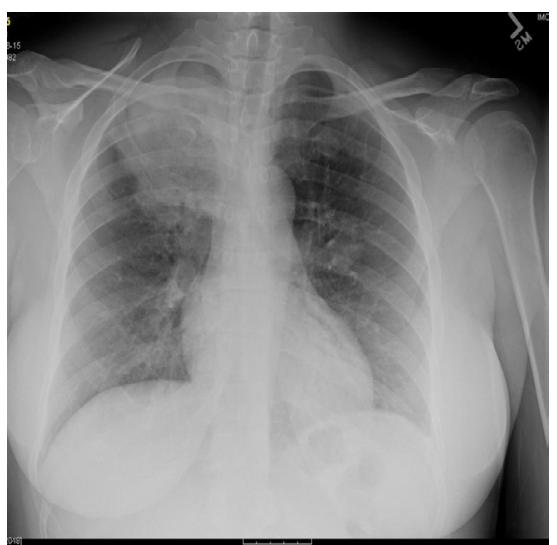

Figure 1: C-XR Anterior-Posterior view showing right upper lobe infiltrate. elevated creatinine $(1.2 \mathrm{mg} / \mathrm{dl})$ and mild transaminitis. A Chest X-ray showed an extensive right upper lung opacification and computed tomography of the thorax revealed a necrotizing right upper lobe infiltrate (Figures 1-4). Broad spectrum antibiotic coverage was started with no clinical improvement and worsening of clinical condition in the next few days. The patient had 3 negative Acid Fast Bacilli (AFB) sputums. Blood and sputum cultures showed no growth while influenza and a respiratory viral panel were negative. Anti-nuclear antibody (ANA) and Cytoplasmic anti-neutrophil cytoplasmic antibody (c-ANCA) were positive. A Bronchoscopy performed showed diffuse friable granular mucosa with no endobronchial lesions (Figure 5). Endobronchial and transbronchial biopsies were performed. AFB and cultures from the BAL were negative. Pathology revealed caseating necrotizing granulomatous inflammation. This combined with c-ANCA positivity made the diagnosis of GPA extremely likely. Patient was treated with pulse steroids and refused cytotoxic therapy and left

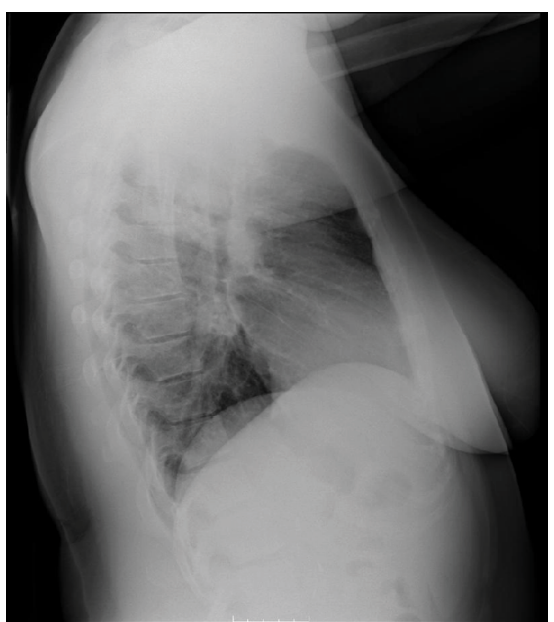

Figure 2: C-XR Lateral view showing upper lobe infiltrate.

*Corresponding author: Kilaru D, Internal Medicine Resident, Nassau University Medical Center, East Meadow, New York, USA, Tel: +5165726501; E-mail: dkilaru@numc.edu

Received August 17, 2017; Accepted August 30, 2017; Published August 31 2017

Citation: Kilaru D, Anand P, Chua W (2017) A Curious Case of Unresolving Pneumonia. J Pulm Respir Med 7: 421. doi: 10.4172/2161-105X.1000421

Copyright: ( 2017 Kilaru D, et al. This is an open-access article distributed under the terms of the Creative Commons Attribution License, which permits unrestricted use, distribution, and reproduction in any medium, provided the original author and source are credited. 


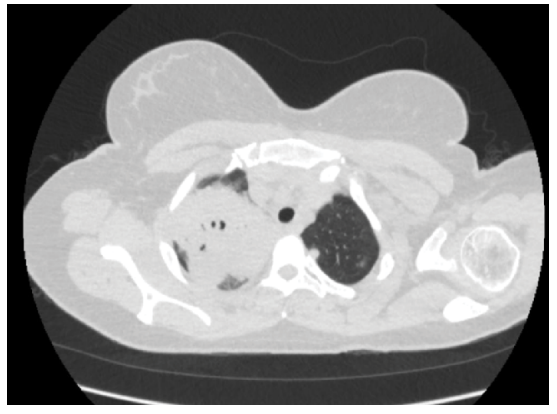

Figure 3: CT thorax showing necrotizing right upper lobe infiltrate.

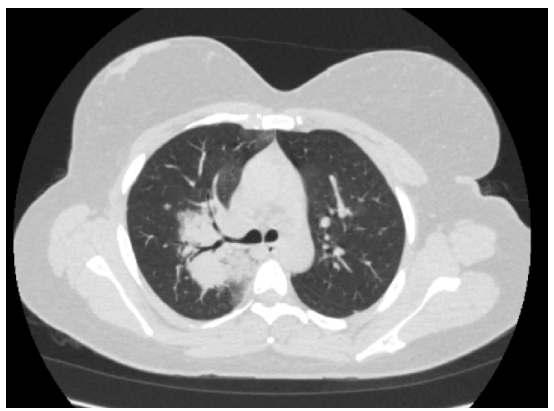

Figure 4: CT thorax showing necrotizing right upper lobe.

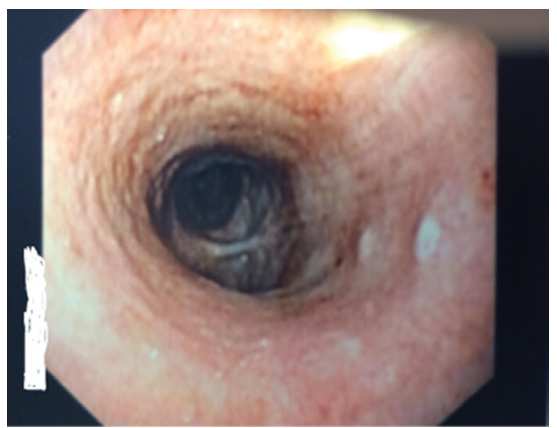

Figure 5: Bronchoscopy showing no endobronchial lesions.

AMA but decided to follow up as an outpatient. Her symptoms and CXR resolved after only steroid treatment alone and she was eventually switched to methotrexate and remained in remission for over a year $[5,6]$.

\section{Discussion}

GPA is characterized by necrotizing granulomatous inflammation and pauci-immune vasculitis in small- and medium-sized blood vessels. It commonly involves the upper and lower respiratory tract and kidneys and can be difficult to distinguish from infectious etiologies and neoplastic diseases given its clinical presentation. C-ANCA is most specific for GPA and is detectable in nearly all patients with active severe GPA. The diagnosis of GPA is generally confirmed with tissue biopsy from a site of active disease, with renal and lung biopsies being more specific. Lung biopsy is typically performed by either open or thoracoscopic lung biopsy. Bronchoscopy is helpful in the evaluation of alveolar hemorrhage however transbronchial biopsy has a low diagnostic yield given the size of the tissue obtained. The mainstay of treatment to induce remission for systemic disease with high dose steroids combined with cytotoxic therapy. However, relapses remain frequent.

\section{Conclusion}

In conclusion, our case demonstrates the broad variability in ANCA associated vasculitis both in presentation and response to different treatments. Our patient had extensive lung disease with systemic symptoms with good response to corticosteroids alone with maintenance of remission for a least a year.

\section{References}

1. Lynch JP, Henry T (2011) Wegener granulomatosis (Granulomatosis with polyangiitis: Evolving concepts in treatment. Semin Respir Crit Care Med 32: 274-297.

2. Comarmond C, Cacoub P (2014) Granulomatosis with polyangiitis (Wegener): Clinical aspects and treatment. Autoimmun Rev 13: 1121-1125.

3. Christopher LT (2016) Granulomatosis with polyangiitis (Wegeners granulomatosis) Herbert SD (Editor). Medscape, New York, USA.

4. Ittikorn S, Nath Z, Pichaya OC, Bhisit C, Dennis TB Jr (2015) Granulomatosis with polyangiitis: A case of nasal mass, necrotic lung, and normal kidneys. Hawaii J Med Public Health 74: 27-29.

5. Roberts KK, Chamberlin MM, Holmes AR, Henderson JL, Hutton RL (2016) Pulmonary microscopic polyangiitis presenting as acute respiratory failure from diffuse alveolar hemorrhage. Roberts. Sarcoidosis Vasc Diffuse Lung Dis 32 372-377.

6. Finkielman JD, Lee AS, Hummel AM, Viss MA, Jacob GL (2007) ANCA are detectable in nearly all patients with active severe Wegener's granulomatosis. Am J Med 120: 643.e9-e14. 